\title{
Illuminating Z-Score in External Quality Assessment for Medical Laboratory
}

\section{Sareen $\mathbf{R}^{*}$}

Department of Pathology, Santokba Durlabhji Memorial Hospital, India

*Corresponding author: Rateesh Sareen, Consultant, Department of Pathology, Santokba Durlabhji Memorial Hospital, 7 Research Center, India, Tel: 01412603401; Email: drrateeshsareen@yahoo.co.in

Received date: October 03, 2018; Accepted date: October 22, 2018; Published date: October 30, 2018

Copyright: () 2018 Sareen R. This is an open-access article distributed under the terms of the Creative Commons Attribution License, which permits unrestricted use, distribution, and reproduction in any medium, provided the original author and source are credited.

\begin{abstract}
The main role of External Quality Assessment (EQA) is to score laboratories on the basis of their performance and provide scores like $Q$ score and $Z$ score to quantitative data to the participant laboratories. These scores are widely applicable for analytical laboratories by scaling difference in the participant's result and assigned value. The present paper deals with interpretation of $Z$ score and provides a clear understanding of the topic.
\end{abstract}

Keywords: Dietary supplements; Micronutrients; Health; Nutrient gaps

\section{Introduction}

External Quality Assessment (EQA) has a pivotal role in quality assurance of medical laboratories. EQA serves as a guiding tool for the participating laboratories which can compare its results for each analyse with those of other participants. It is extremely important for participating laboratories to understand the EQA report, assigned values, and performance evaluation so that appropriate steps can be taken in case of outlier results. The paper focuses on commonly used terminology $\mathrm{Z}$ score. External quality assessment (EQA) was first introduced in the 1950s and 1960s in response to the growing role of laboratory testing as an essential part of disease diagnosis and management and an awareness of the extent of variability in results from one laboratory to another, for example as described by Mitchell Lewis following his initial inter laboratory trials in the UK [1].

EQA is nowadays available to some degree in all developed healthcare systems and is a means to improving the quality of laboratory performance [2]. The World Health Organization updated and re-issued its manual for the establishment and operation of an EQA programme in 2016, providing comprehensive guidance on the strategies and responsibilities for EQA operation, and the international standards ISO17043:2010 Conformity assessment-General requirements for proficiency testing and ISO13528:2015 Statistical methods for use in proficiency testing by interlaboratory comparison give details of the quality standards directly applicable to EQA providers [3]. The European Quality Assurance in Laboratory Medicine (EQALM) organization is the preeminent professional association dedicated solely to EQA provision [4]. Organisations such as EQALM give EQA providers the opportunity to share best practice and learn from the experience of others, with the objective that an appropriate and effective performance standard is applied to laboratory assessment, wherever the test may be undertaken. Harmonisation and standardization of laboratory medicine practice is not only desirable as part of good patient care but becomes essential when patients' samples may be examined in different locations or by different healthcare providers.
EQA of the analytical phase of diagnostic testing entails the EQA provider distributing a sample or other artefact of known but undisclosed content to the participating laboratories or testing sites, which are usually medical laboratories but are increasingly to be found in 'point of care' situations, such as the ward or operating theatre, in clinics or doctors' surgeries and in the community. The sites test the material as if it were a patient's sample and report their results to the EQA provider for statistical analysis. By comparison with minimum acceptable performance limits, the EQA provider can identify laboratories, methods, kits or reagents with out-of-consensus results and ensure that appropriate corrective action is taken, either by the laboratory or some other regulatory agency. EQA for point-of-care testing (POCT), especially when outside the hospital environment, has different requirements from testing provided by laboratory professionals, although immediate clinical decisions may be made according to the results [4].

The standard for methods for statistical analysis in EQA are described in ISO 13528: 2015 but the standard can be applied in a number of forms. Although the principles of statistical use in EQA is relatively straightforward, requiring the definition of a target value and statistical performance limits based on distance from the target, there are a number of statistical processes and manipulations involved that require some expert knowledge on the part of the EQA provider and translation into an intelligible format for participants' reports $[5,6]$. The challenge of EQA provision in resource limited countries and the implementation of regional proficiency testing in East Africa, is extensively and comprehensively reviewed by Jane Carter, Stephen Munene and co-workers. Their work represents a truly global summary of the impact and influence of EQA in a challenging environment where allocation of resources to laboratory testing is unfortunately still of very low priority [7].

\section{Q Score}

The term 'Q score' was first of all used by Jack Landis in 1963 [8]. It was initially used as a metric to determine the familiarity of a brand or celebrity and measured the appeal of each among people familiar with the entity being measured. The laboratory Q score refers to the comparison of test results of analyte with the acceptability ranges. It 
consists of relative difference between the value reported by the laboratory and the assigned value.

$$
\begin{aligned}
& Q \text { score }=\text { Reported value }- \text { Assigned Value } \\
& \text { /Assigned Value }
\end{aligned}
$$

\section{Z score}

It is the difference between the value reported by the laboratory and the assigned value corrected for variability [9].

\section{$Z$ score $=$ Reported value - Assigned Value / Standard deviation}

The participant's result is converted into $Z$ score as per recommendation of 1993 harmonized protocol [10]. Say if the participant's result is $x_{a}$

Then, according to equation

$Z=(x-x a) / ?^{\wedge}$

Where,

$\mathrm{X}$ : Assigned value - True value of the concentration of analyte in PT

$\mathrm{x}_{\mathrm{a}}$ : Participant value

$\sigma^{\wedge}$ : Fitness for purpose based 'Standard deviation' for PT

$\mathrm{Z}$ score makes proficiency test score immediately apparent to the observer irrespective of the concentration, identity, physical property and EQA scheme provider. The interpretation of $Z$ score is based on assumed model based on the scheme provider's fitness for purpose criterion which is represented by the standard deviation for proficiency assessment, assuming that the assigned value is very close to true value so that $\mathrm{Z}$ score follows normal distribution.

The following interpretation helps in decoding ' $Z$ ' score:

$\mathrm{Z}$ score zero: It implies a perfect result. It happens rarely even with the most competent laboratory [11].

$\mathrm{Z}$ score between -2 to +2 : These scores are designated 'acceptable' or 'satisfactory'.

$\mathrm{Z}$ score outside range -3 to +3 is very unusual, regarded as 'unsatisfactory' or 'not acceptable'. It requires remedial action.

$\mathrm{Z}$ score between -2 to -3 and +2 to +3 of isolated event are regarded as 'questionable'.

These criteria are stated in ISO/IEC standard 17043:2010 [11]. The performance of the individual laboratory is compared against the dispersion of results obtained by the participants in the peer-group in each survey. One must not forget that these limits are variable and may change with time as methods and instruments evolve. The limits may vary between peer-groups measuring the same component as they are statistical based criteria. Imprecise-method peer groups will have a large acceptance interval whereas precise-method peer groups will have a small interval for acceptable results, independent of what is required for clinical needs.

In conditions where there are lack of universally applicable fitness for purpose criterion like when participants carry out daily routine work, it would be prudent on the part of providers, not to provide scores, rather give an assigned value (with uncertainty) and laboratory error. This is sometimes referred as relative error or simply ' $Q$ Score' [10]. In case of using such scores it is advisable that the individual participants have their own criterion for fitness for purpose. It is for the provider to clearly indicate as 'for informal use only', caption in results so that incorrect judgments based on scores are curtailed [12]. PT providers, participants and end users should avoid classification and ranking of the laboratories on the basis of their ' $\mathrm{Z}$ score' as it creates confusion. Interpretation of $Z$ score relies on the idea that, if all laboratories performed similarly and in accordance with the requirements set, their work would be approximately normally distributed. So actually it is not the individual laboratory performance rather idealized performance by participant laboratories [6]. Thus over a time period Z score compares the participant with PT providers' criterion of good performance [4]. The long term view of the participant laboratory can be best demonstrated by plotting successive $\mathrm{Z}$ scores on a control chart based on a zero mean and unit standard deviation [10]. Single round of PT or from a single analyst should not be used as the sole mean of analyst performance [11]. PT is one of the components of quality in laboratory [5]. All PT failures should be investigated individually and corrective action initiated [10]. Therefore, proper and timely evaluation of EQA survey reports is essential and evens a must for accreditation [10].

\section{References}

1. Lewis SM, Burgess BJ (1969) Quality control in haematology: Report of interlaboratory trials in britain. BMJ 4: 253-256.

2. Thompson M, Wood R (2006) The International Harmonized Protocol for the proficiency testing of (chemical) analytical laboratories. Pure Appl Chem 65: 2123-2144.

3. WHO manual for organizing a national external quality assessment programme for health laboratories and other testing sites.

4. Stavelin SA, Albe X, Meijer P, Sarkany E, Finlay MacKenzie F (2017) EQALM: An overview of the European Organization for External Quality Assurance Providers in Laboratory Medicine (EQALM). Biochem Med 27:30-6.

5. Coucke W (2017) Demystifying EQA statistics and reports. Biochem Med 27: 37-48.

6. Kristensen GB, Meijer P (2017) Interpretation of EQA results and EQAbased trouble shooting. Biochem Med 27: 49-62.

7. Carter JY (2017) External quality assessment in resource limited countries. Biochem Med 27: 97-109.

8. Bialik, Carl (2018) "The Numbers Behind Modern Star Search". The Wall Street Journal.

9. Analytical Methods Committee (1995) Uncertainty of measurementimplications for its use in analytical science. Analyst 120: 2303-2308.

10. Thompson M, Stephen. Ellison R, Wood R (2006) The International harmonized protocol for the proficiency testing of analytical chemistry laboratories Pure Appl. Chem 78: 145-196.

11. International Organization for Standardization/International Electrotechnical Commission. Conformity assessment-General requirements for proficiency testing. ISO 17043. Geneva: ISO/IEC.

12. Fearn T, Fisher S, Thompson M, Ellison SLR (2002) "A decision theory approach to fitness-for purpose in analytical measurement", Analyst 127: 818-824. 